\title{
Radiation Driven Implosion and Triggered Star Formation
}

\author{
T. G. Bisbas ${ }^{1}$, A. P. Whitworth ${ }^{2}$, R. Wünsch ${ }^{1}$, D. A. Hubber ${ }^{3}$, \\ and S. Walch ${ }^{2}$ \\ ${ }^{1}$ Astronomical Institute, Academy of Sciences of the Czech Republic, Boční II 1401, 14131 \\ Prague, Czech Republic. Email: t.bisbas@astro.cf.ac.uk \\ ${ }^{2}$ School of Physics and Astronomy, Cardiff University, Queens Buildings, The Parade, Cardiff, \\ CF24 3AA, United Kingdom \\ ${ }^{3}$ Department of Physics and Astronomy, University of Sheffield, Hicks Building, Hounsfield \\ Road, Sheffield S3 7RH, United Kingdom
}

\begin{abstract}
We present simulations of stable isothermal clouds exposed to ionizing radiation from a discrete external source, and identify the conditions that lead to Radiatively Driven Implosion and Star Formation. We use the Smoothed Particle Hydrodynamics code SEREN (Hubber et al. 2010) and the HEALPix-based photoionization algorithm described in Bisbas et al. (2009). We find that the incident ionizing flux is the critical parameter determining the evolution; high fluxes disperse the cloud, whereas low fluxes trigger star formation. We find a clear connection between the intensity of the incident flux and the parameters of star formation.
\end{abstract}

Keywords. hydrodynamics, methods: numerical, stars: formation, (ISM:) HII regions

\section{Introduction}

When an expanding HiI region overruns a pre-existing cloud, it compresses it by driving an ionization front and a shock wave into it (Sandford et al. 1982; Bertoldi 1989; Lefloch \& Lazareff 1994). The inner parts may become gravitationally unstable and collapse to form new stars. This mechanism is known as Radiation Driven Implosion (RDI). Observations (Lefloch \& Lazareff 1995; Lefloch et al. 1997; Sugitani et al. 1999, 2000; Ikeda et al. 2008; Morgan et al. 2008; Chahuan et al. 2009) strongly support a connection between the RDI mechanism and the formation of Young Stellar Objects (YSO). Simulations of the interaction of ultraviolet ionizing radiation with self-gravitating clouds have been presented by various authors (Kessel-Deynet \& Burkert 2003; Esquivel \& Raga 2007; Gritschneder et al. 2009; Miao et al. 2009). However, no model can explain where star formation takes place (in the core or at the periphery) or when (during the maximum compression phase or earlier - Deharveng et al. 2005). The aim of this work is to answer questions of whether the ionizing radiation incident upon stable clouds is able to trigger the formation of new stars or not, and how the process and the properties of this star formation are connected with the intensity of the incident flux. In Section 2 we give a brief description of the numerical treatment and the initial conditions we use. In Section 3 we discuss the results of our simulations. We summarize in Section 4.

\section{Numerical Treatment and Initial Conditions}

We use the Smoothed Particle Hydrodynamics (SPH) code SEREN†, fully described in Hubber et al. (2010), with an ionization routine (Bisbas et al. 2009) based on the 
HEALPix $\ddagger$ sphere tesselation code (Górski et al. 2005). We use a barotropic equation of state (i.e. Bonnell 1994) to set the temperature of the neutral gas as $T_{\mathrm{N}}(\rho)=$ $T_{\text {IS }}\left\{1+\left(\rho / \rho_{\text {C R IT }}\right)^{\gamma-1}\right\}$, where $T_{\text {ISo }}=10 \mathrm{~K}, \rho_{\text {CR IT }}=10^{-13} \mathrm{~g} \mathrm{~cm}^{-3}$ and $\gamma=5 / 3$ is the ratio of specific heats. The temperature of the ionized gas is set to $T_{\mathrm{i}}=10^{4} \mathrm{~K}$, except in the transition zone between the two extremes, where it changes smoothly from $T_{\mathrm{i}}$ to $T_{\mathrm{N}}$ (see Bisbas et al. 2009). We include sink particles (Bate et al. 1995) with radii $R_{\mathrm{SINK}}=2.5 \mathrm{AU}$ created if $\rho>\rho_{\mathrm{SINK}}=10^{-11} \mathrm{~g} \mathrm{~cm}^{-3}$.

Our clouds are stable Bonnor-Ebert spheres (heareafter 'BES') with dimensionless cutoff radii $\xi_{\mathrm{B}}=4,5,6$ (see Bonnor 1956 for its definition) and with masses $M=2,5,10 \mathrm{M}_{\odot}$. The particle resolution we use is $5 \times 10^{4} \mathrm{SPH}$ particles per solar mass (cf. Hubber et al. 2006). We use a single source emitting Lyman- $\alpha$ photons. We place the BESs at distance $D=10 R$ from the ionizing source, where $R$ is the radius of the cloud (in pc), in order to keep constant the divergence of the incident flux and as parallel as possible. We run simulations with a wide range of emission rates $\dot{\mathcal{N}}_{\mathrm{LyC}}=10^{x} \mathrm{~s}^{-1}$, where $x=48,48.5, \ldots 52$.

\section{Results}

In Fig.1a we present a semi-logarithmic diagram where we correlate the intensity of the incident ionizing flux with the initial mass of each BES. The lines define subsets of parameter space where models either show star formation (left) or not (right), with accuracy 0.25 dex. It can be seen that as the mass of the BES decreases (and as a result $\xi_{\mathrm{B}}$ increases) the clouds appear to dissolve in higher fluxes. This is because for a given $\xi_{\mathrm{B}}$, the density $\rho_{\mathrm{c}}$ at the centre of each BES increases with decreasing $M$.

We also find that the Strømgren radius at the end of the $R$-type expansion determines whether stars are formed or not; if the ionization front has not overrun the central core of the BES, then the incident flux will trigger star formation during the $D$-type expansion of the HII region. In the opposite case there is not enough material to undergo gravitational collapse and form stars.

The time, $t_{\mathrm{SINK}}$, between the beginning of the $D$-type expansion and the first sink creation (beginning of star formation) increases with decreasing ionizing flux. This finding is in agreement with simulations of the RDI performed by Gritschneder et al. (2009). Figure $1 \mathrm{~b}$ is a logarithmic diagram where we plot the values of $t_{\mathrm{SINK}}$ versus the incident flux $\Phi_{D}$. Remarkably, it can be seen that $t_{\mathrm{SINK}}$ does not depend on $\xi_{\mathrm{B}}$. Results from our simulations can be described with a power law of the form $t_{\mathrm{SINK}}=80 \times \Phi_{\mathrm{D}}^{-0.3}\left(t_{\mathrm{SINK}}\right.$ in Myr, $\Phi_{\mathrm{D}}$ in $\left.\mathrm{cm}^{-2} \mathrm{~s}^{-1}\right)$.

Figure 2 shows column density plots of a BES with $M=10 \mathrm{M}_{\odot}$ and with $\xi_{\mathrm{B}}=6$ at $t_{\mathrm{SINK}}$ for different fluxes. A common feature in all our simulations is that stars form close to the symmetry axis joining the centre of the cloud to the exciting star. This is probably a consequence of the initial spherical symmetry of the cloud and it is in an agreement with observations by Sugitani et al. (1999). The distance $d_{\mathrm{t}}$ between the first sink particle and the ionization front is a function of the ionizing flux and the BES parameters (see Fig.1c where we plot $d_{\mathrm{t}} / 2 R$ for all BESs with $\left.\xi_{\mathrm{B}}=6\right)$. We find that for low fluxes stars tend to form away from the periphery, whereas for high fluxes stars tend to form at the periphery of the cloud. Similar results are found also with $\xi_{\mathrm{B}}=4$ and $\xi_{\mathrm{B}}=5$.

Figure 2 shows that the lateral compression, $w_{\mathrm{d}}$, of the BESs at the beginning of star formation is connected to the intensity of the incident flux. We see that for low fluxes, $w_{\mathrm{d}}$ is quite high and the cloud has a $\mathbf{U}$-shape structure, whereas for high fluxes $w_{\mathrm{d}}$ is small and the cloud has a V-shape structure. In Fig.1d we plot $w_{\mathrm{d}} / 2 R$ for all BESs with $\xi_{\mathrm{B}}=6$ 
and we find that stars tend to form during maximum compression once the incident flux is increased. Similar results are found also for the rest of the clumps.

\section{Conclusions}

We present simulations of RDI in stable clouds represented by BESs. We performed 75 simulations with clouds of different masses, different dimensionless radii, and with a wide range of incident fluxes. In general we find a connection between the incident ionizing flux and whether the cloud is induced to form stars or not by the flux. Our results only apply to clouds which have similar density structures.

We introduce a semi-logarithmic diagram (flux-mass diagram) where we correlate the intensity of the incident flux and the initial mass of each BES, and we define zones of Star Formation and no-Star Formation. We find that if the Strømgren radius has not overrun the central core of the BES by the end of the $R$-type expansion, the ionizing radiation will trigger star formation. The time when star formation occurs increases with decreasing flux, and does not depend on $\xi_{\mathrm{B}}$. A power-law of the form $t_{\mathrm{SINK}}=80 \times \Phi_{\mathrm{D}}^{-0.3}$ fits very well with the results of our models. Finally, as the incident flux increases, stars tend to form closer to the periphery of the cloud and during its maximum compression phase.
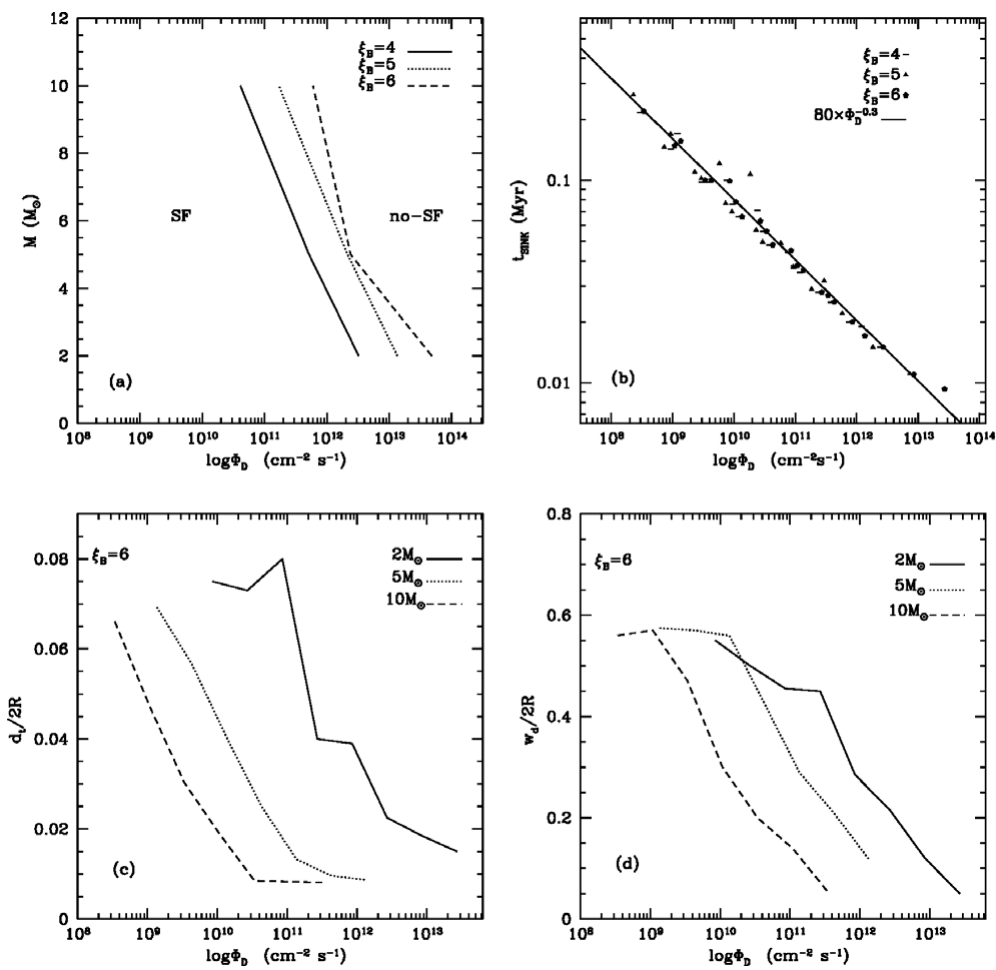

Figure 1. (a) The flux-mass semi-logarithmic diagram where we define areas where stars are formed (SF) and areas where stars are not formed (no-SF) depending on the dimensionless radius $\xi_{\mathrm{B}}$ of a BES. (b) Logarithmic diagram of the incident flux versus $t_{\mathrm{SINK}}$. The power law we propose (solid line) fits very well with our simulations $\left(t_{\mathrm{SINK}}\right.$ is in Myr and $\Phi_{\mathrm{D}}$ is in $\left.\mathrm{cm}^{-2} \mathrm{~s}^{-1}\right)$. (c) Star formation occurs at the periphery with increasing flux. (d) Star formation occurs during maximum compression with increasing flux. 


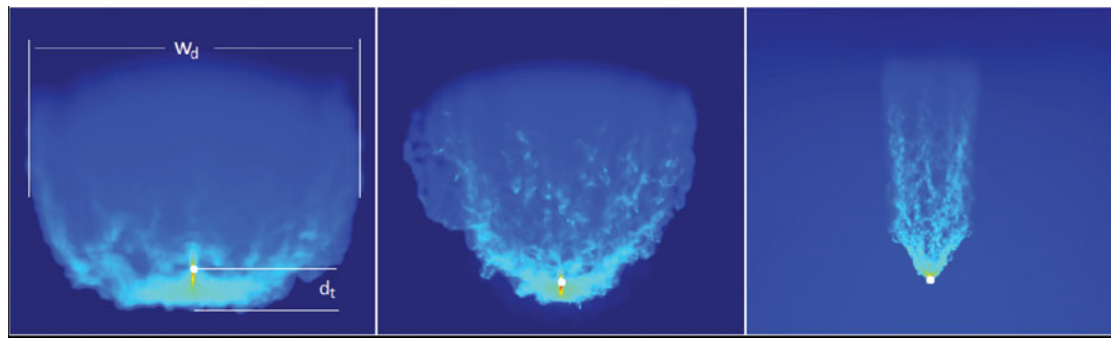

Figure 2. Column density plots of a BES with $M=10 \mathrm{M}_{\odot}$ and $\xi_{\mathrm{B}}=6$ at $t_{\mathrm{SINK}}$ when it is exposed to three different intensities of flux (flux increases from left to right). The white dots are sink particles. In the left plot we draw the values of $d_{\mathrm{t}}$ and $w_{\mathrm{d}}$.

Acknowledgments: TGB and RW acknowledge support from the project LC06014Centre for Theoretical Astrophysics of the Ministry of Education, Youth and Sports of the Czech Republic. APW and SW gratefully acknowledge the support of the Marie Curie Research Training Network CONSTELLATION (Ref. MRTN-CT-2006-035890). DAH is funded by a Leverhulme Trust Research Project Grand (F/00 118/BJ). The computations in this work were carried out on Merlin Supercomputer of Cardiff University. The column density plots were made using the SPLASH visualization code (Price 2007). The authors acknowledge the anonymous referee for the useful comments.

\section{References}

Barnes, J. \& Hut, P. 1986, Nature, 324, 446

Bate, M. R., Bonnell, I. A., \& Price, N. M. 1995, MNRAS, 277, 362

Bertoldi, F. 1989, ApJ, 346, 735

Bisbas, T. G., Wünsch, R., Whitworth, A. P., \& Hubber, D. A. 2009, A\&A, 497, 649

Bonnell, I. A. 1994, MNRAS, 269, 837

Bonnor, W. B. 1956, MNRAS, 116, 351

Chauhan, N., Pandey, A. K., Ogura, K., Ojha, D. K., Bhatt, B. C., Ghosh, S. K., \& Rawat, P. S. 2009, MNRAS, 396, 964

Esquivel, A. \& Raga, A. C. 2007, MNRAS, 377, 383

Deharveng, L., Zavagno, A., \& Caplan, J. 2005, A\&A, 433, 565

Górski, K. M., Hivon, E., Banday, A. J., Wandelt, B. D., Hansen, F. K., Reinecke, M., \& Bartelmann, M. 2005, ApJ, 622, 759

Gritschneder, M., Naab, T., Burkert, A., Walch, S., Heitsch, F., \& Wetzstein, M. 2009, MNRAS, 393, 21

Hubber, D. A., Batty, C. P., McLeod, A., \& Whitworth, A. P., 2010, A\& A, submitted

Hubber, D. A., Goodwin, S. P., \& Whitworth, A. P. 2006, A\&SA, 450, 881

Ikeda, H., et al. 2008, AJ, 135, 2323

Kessel-Deynet, O. \& Burkert, A. 2003, MNRAS, 338, 545

Lefloch, B. \& Lazareff, B. 1994, A $\mho A, 289,559$

Lefloch, B. \& Lazareff, B. 1995, A\&A, 301, 522

Lefloch, B., Lazareff, B., \& Castets, A. 1997, A\& A, 324, 249

Miao, J., White, G. J., Thompson, M. A., \& Nelson, R. P. 2009, ApJ, 692, 382

Monaghan, J. J. 1992, ARAA, 30, 543

Morgan, L. K., Thompson, M. A., Urquhart, J. S., \& White, G. J. 2008, A\& $A, 477,557$

Price, D. J. 2007, Publications of the Astronomical Society of Australia, 24, 159

Sandford, M. T., II, Whitaker, R. W., \& Klein, R. I. 1982, ApJ, 260, 183

Sugitani, K., Tamura, M., \& Ogura, K. 1999, Star Formation 1999, Proceedings of Star Formation 1999, held in Nagoya, Japan, June 21 - 25, 1999, Editor: T. Nakamoto, Nobeyama Radio Observatory, p. 358-364, 358

Sugitani, K., Matsuo, H., Nakano, M., Tamura, M., \& Ogura, K. 2000, AJ, 119, 323 